\title{
Chemical bonding and optical bowing in III-nitrides solid solutions
}

\author{
O.V. Voznyy, V.G. Deibuk \\ Chernivtsi national university, 2 Kotsyubinskiy Str., 58012 Chernivtsi, Ukraine \\ E-mail:voznyy@elegance.cv.ua,vdei@chnu.cv.ua
}

\begin{abstract}
Chemical bonding and electronic properties of III-nitrides solid solutions are studied using a model empirical pseudopotential method in modified virtual crystal approximation taking into account positional and compositional disorder. The changes in chemical bonding are analyzed in different ionicity models. The bowing is mainly caused by compositional disorder, volume deformation effects and valence charge redistribution in the alloys. The connection between the value of the bowing and basic parameters of the materials is found.
\end{abstract}

Keywords: III-nitrides, chemical bonding, ionicity, bowing, empirical pseudopotential, disordered alloys.

Paper received 21.03.03; accepted for publication 16.06.03.

\section{Introduction}

The group-III wurtzite nitrides GaN, AlN, InN and their solid solutions have direct energy gaps, high ionicity, short bond lengths and high stability which makes them useful materials for high power and high frequency transistors [1] and also for optoelectronic devices operating in a wide spectrum of wavelengths [2].

Solid solutions are used in III-nitrides heterostructure devices to avoid the formation of misfit dislocations in the layers due to large lattice constant differences that arise between pure compounds [1,2]. Solid solutions allow obtaining the needed band gap for a given lattice constant; particularly one can find some ternary alloy compositions with the same band gap. It is well known that physical properties of the alloys exhibit nonlinear dependencies on composition. That is why a theoretical model predicting the properties of the alloys is needed.

Chemical bonding determines the majority of materials physical properties. In this paper we study the influence of chemical bonding rebuilding on electronic properties of III-nitrides. The analysis of chemical bonding is made in different models, which were successfully used to describe mechanical and elastic properties of different materials. The investigation of III-nitrides alloys was held out taking into account positional and compositional disorder.

\section{Calculation method}

To describe the chemical bonding Philips proposed an ionicity model based on empirical data for optical and homopolar gaps [3]. One of the most remarkable features of Philips's ionicity scale is the existence of a critical value of $f_{i}$ that separates fourfold-coordinated from sixfold-coordinated compounds.

The other parameter that describes chemical bonding is polarity $a_{p}$ [4], which reflects the asymmetry of the pseudopotential:

$$
\alpha_{p}=-\frac{V_{3}^{A}}{V_{3}^{S}},
$$

where $V_{3}{ }^{A}$ and $V_{3}{ }^{S}$-antisymmetric and symmetric pseudopotential form-factors at $G=\frac{2 \pi}{a_{\text {cub }}}(111)=\frac{\sqrt{2} \pi}{a_{\text {hex }}}(111)$. 


\section{O.V. Voznyy, V.G. Deibuk: Chemical bonding and optical bowing in III-nitrides ...}

Garcia and Cohen [5] offered a first-principles ionicity scale based on charge distribution asymmetry. They decompose charge density into symmetric and antisymmetric components and construct their Fourier components:

$$
\begin{aligned}
& \rho_{S}(G)=\frac{1}{2}\left[\rho(G)+\rho^{*}(G)\right] \\
& \rho_{A}(G)=\frac{1}{2}\left[\rho(G)-\rho^{*}(G)\right]
\end{aligned}
$$

To describe quantitatively the chemical bonding the charge asymmetry coefficient is used:

$$
g=\sqrt{\frac{S_{A}}{S_{S}}}
$$

where $S_{S}$ and $S_{A}$ - the integrated quantities which measure the strength of the symmetric and antisymmetric components of over the charge density over the whole unit cell:

$$
\begin{aligned}
& S_{S}=\sum_{G}\left|\rho_{S}(G)\right|^{2}=\frac{1}{\Omega} \int_{\Omega} \rho_{S}^{2}(r) d r \\
& S_{A}=\sum_{G}\left|\rho_{A}(G)\right|^{2}=\frac{1}{\Omega} \int_{\Omega} \rho_{A}^{2}(r) d r
\end{aligned}
$$

We use three mentioned parameters to analyze chemical bonding of III-nitrides solid solutions.

The calculations of the electronic structures and valence charge densities were made using the model empirical pseudopotential method [6,7]. We used functional form of the pseudopotential, which is continuous in reciprocal space and depends on local strain to describe the charge density redistribution and pseudopotential screening due to changes of the local atomic environment [8]:

$$
v_{\alpha}(\mathbf{k}, \varepsilon)=a_{0 \alpha} \frac{\left(\mathbf{k}^{2}-a_{1 \alpha}\right)}{a_{2 \alpha} e^{a_{3 \alpha} \mathbf{k}^{2}}-1}\left[1+a_{4 \alpha} \operatorname{Tr}(\varepsilon)\right],
$$

The fitting parameters $a_{i}$ were taken from the approximation of the ab initio norm-conserving Hamman pseudopotentials [9], screened by Levine and Louie dielectric function [10]. Then the parameters were adjusted to reproduce the experimental values of the interband transitions energies at high-symmetric points of Brillouin zone (Table 1). To take the deformations into account cor-

\begin{tabular}{|c|c|c|c|}
\hline Transition & Energy, eV This work & Energy, eV & Other calculations and experiments \\
\hline \multirow[t]{6}{*}{ GaN } & $\Gamma_{6 \mathrm{~V}^{-}} \Gamma_{1 \mathrm{C}}\left(E_{g}\right)$ & 3.5 & $3.507^{\mathrm{a}}, 3.5^{\mathrm{b}}, 3.51^{\mathrm{c}}$ \\
\hline & $\Gamma_{3 \mathrm{~V}^{-}} \Gamma_{6 \mathrm{~V}}($ top valence band width $)$ & 7.5 & $6.8^{\mathrm{b}}, 7.4(8)^{\mathrm{d}}$ \\
\hline & $\Gamma_{1 \mathrm{~V}^{-}} \Gamma_{6 \mathrm{~V}}\left(\Delta_{\mathrm{cr}}\right)$ & 0.01 & $0.019^{\mathrm{a}}, 0.02^{\mathrm{b}}, 0.038^{\mathrm{c}}$ \\
\hline & $\mathrm{L}_{2,4 \mathrm{~V}}-\mathrm{L}_{1,3 \mathrm{C}}$ & 7.26 & $7.57^{\mathrm{b}}, 6.4(8.2)^{\mathrm{d}}$ \\
\hline & $\mathrm{M}_{4 \mathrm{~V}}-\mathrm{M}_{3 \mathrm{C}}$ & 7.6 & $7.7^{\mathrm{b}}, 7.6^{\mathrm{d}}$ \\
\hline & $\mathrm{H}_{3 \mathrm{~V}}-\mathrm{H}_{3 \mathrm{C}}$ & 7.9 & $9^{\mathrm{b}}, 8.1(9.9)^{\mathrm{d}}$ \\
\hline \multirow[t]{6}{*}{ AlN } & $\Gamma_{6 \mathrm{~V}^{-}} \Gamma_{1 \mathrm{C}}\left(E_{g}\right)$ & 6.23 & $6.23^{\mathrm{a}}, 6.24^{\mathrm{c}}$ \\
\hline & $\Gamma_{3 \mathrm{~V}}-\Gamma_{6 \mathrm{~V}}($ top valence band width $)$ & 6.57 & $6.1(6.9)^{\mathrm{d}}$ \\
\hline & $\Gamma_{1 \mathrm{~V}^{-}} \Gamma_{6 \mathrm{~V}}\left(\Delta_{\mathrm{cr}}\right)$ & -0.22 & $-0.164^{\mathrm{a}},-0.17^{\mathrm{c}}$ \\
\hline & $\mathrm{L}_{2,4 \mathrm{~V}}-\mathrm{L}_{1,3 \mathrm{C}}$ & 9.9 & $8.6^{\mathrm{d}}$ \\
\hline & $\mathrm{M}_{4 \mathrm{~V}}-\mathrm{M}_{3 \mathrm{C}}$ & 10.34 & $8.5^{\mathrm{d}}$ \\
\hline & $\mathrm{H}_{3 \mathrm{~V}}-\mathrm{H}_{3 \mathrm{C}}$ & 10.56 & $10.5^{\mathrm{d}}$ \\
\hline \multirow[t]{6}{*}{ InN } & $\Gamma_{6 \mathrm{~V}^{-}} \Gamma_{1 \mathrm{C}}\left(E_{g}\right)$ & 0.69 & $0.7^{\mathrm{e}}, 2.04^{\mathrm{b}}$ \\
\hline & $\Gamma_{3 \mathrm{~V}^{-}} \Gamma_{6 \mathrm{~V}}($ top valence band width $)$ & 6 & $5.77^{\mathrm{b}}$ \\
\hline & $\Gamma_{1 \mathrm{~V}^{-}} \Gamma_{6 \mathrm{~V}}\left(\Delta_{\mathrm{cr}}\right)$ & 0.04 & $0.041^{\mathrm{a}}, 0.017^{\mathrm{b}}, 0.06^{\mathrm{c}}$ \\
\hline & $\mathrm{L}_{2,4 \mathrm{~V}}-\mathrm{L}_{1,3 \mathrm{C}}$ & 5.2 & $5.83^{\mathrm{b}}$ \\
\hline & $\mathrm{M}_{4 \mathrm{~V}}-\mathrm{M}_{3 \mathrm{C}}$ & 5.2 & $5.8^{\mathrm{b}}$ \\
\hline & $\mathrm{H}_{3 \mathrm{~V}}-\mathrm{H}_{3 \mathrm{C}}$ & 5.97 & $6.5^{b}$ \\
\hline
\end{tabular}
rectly, the values of the deformation potentials were included in the fitting procedure:

$a_{g}=V \frac{\partial E_{g}}{\partial V}$

Table 1. Energies of the interband transitions in high-symmetry points.

\footnotetext{
a recommended values [11]

$\mathrm{b}$ empirical pseudopotentail method [12]

${ }^{c}$ first-principles and empirical pseudopotential method calculations [20]

$\mathrm{d}$ first-principles calculations with and without GW corrections [21]

e GaInN experiment [25]
} 


\section{O.V. Voznyy, V.G. Deibuk: Chemical bonding and optical bowing in III-nitrides ...}

We used the recommended values of the deformation potentials from [11]: $a_{g}(\mathrm{GaN})=-8.2 \mathrm{eV}, a_{g}(\mathrm{AlN})=$ $=-9 \mathrm{eV}, a_{g}(\mathrm{InN})=-4 \mathrm{eV}$. The final values of $a_{i}$ are presented in Table 2. Obtained pseudopotentials are in good agreement with the form-factors from other works [12] (Fig. 1).

The basis of 263 plane waves was used in calculations, which corresponds to $14 \mathrm{Ry}$ energy cut-off. The integration over the Brillouin zone was held using the Chadi-Cohen special points set [13].

In the alloys the atoms of different species are randomly distributed breaking in such a way the translational symmetry of the crystal. Moreover the atomic positions in the alloy shift from the ideal positions given by Vegard's rule due to difference of the bond lengths of the alloy components.

Such features can be taken into account by using the density functional theory considering supercells containing thousands of atoms and though such calculations are very time consuming. The typical approaches to the study of alloys are based on approximations in which all possible, inequivalent, microscopic configurations are averaged into an effective medium having the same crystal

Table 2. Parameters of the screened atomic pseudopotentials in GaN, AIN, InN (in atomic units).

\begin{tabular}{lcllll}
\hline Atom & $a_{0}$ & $a_{1}$ & $a_{2}$ & $a_{3}$ & $a_{4}$ \\
\hline $\mathrm{Ga}$ & 7.247 & 1.66 & 1.403 & 0.178 & 1.67 \\
\hline $\mathrm{N}$ in GaN & 25.2 & 4.68 & 2.61 & 0.346 & - \\
\hline $\mathrm{Al}$ & 7 & 2 & 1.5 & 0.18 & 2.2 \\
\hline $\mathrm{N}$ in $\mathrm{AlN}$ & 53 & 4.72 & 4.13 & 0.35 & - \\
\hline $\mathrm{In}$ & 11.1 & 1.176 & 1.35 & 0.266 & 1.32 \\
\hline $\mathrm{N}$ in $\mathrm{InN}$ & 45.9 & 5.14 & 4.87 & 0.425 & - \\
\hline \hline
\end{tabular}

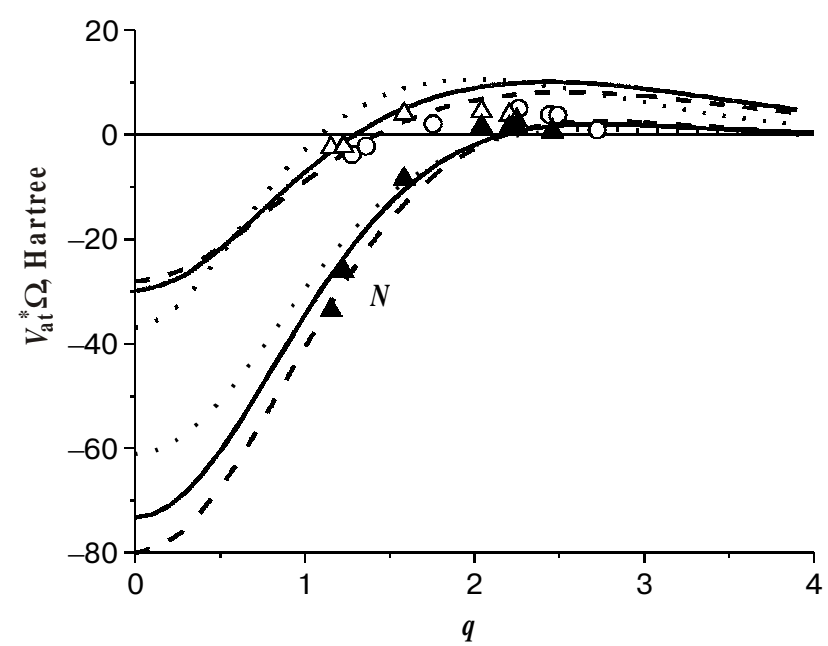

Fig. 1. The screened atomic pseudopotentials for $\mathrm{Ga}$ and $\mathrm{N}$ in GaN (solid line), $\mathrm{Al}$ and $\mathrm{N}$ in $\mathrm{AlN}$ (dashed line), In and $\mathrm{N}$ in $\mathrm{InN}$ (dotted line) plotted in reciprocal space. Form-factors from [12]: circles - Ga in GaN, triangles - In and $\mathrm{N}$ in $\mathrm{InN}$. structure as the underlying lattice, in order to recover the translational symmetry.

We used the virtual crystal approximation (VCA) to study the alloys. The compositional disorder was considered in a second order of perturbation theory [10, 14]. For each energy eigenvalue the corrections due to the presence of a nonperiodic potential due to chemical disorder were calculated:

$$
\begin{aligned}
& E_{n}(\mathbf{k}, x)=E_{n}^{(0)}(\mathbf{k}, x)+x(1-x) \times \\
& \times \sum_{n^{\prime} k^{\prime}} \frac{k n, \mathbf{k}, x|\Delta V| n^{\prime}, \mathbf{k}^{\prime}, x>\left.\right|^{2}}{E_{n}^{(0)}(\mathbf{k}, x)-E_{n^{\prime}}^{(0)}\left(\mathbf{k}^{\prime}, x\right)}
\end{aligned}
$$

where $E_{n}{ }^{(0)}(\boldsymbol{k}, x)-n$-th band energy at $\boldsymbol{k}$ point in the reciprocal space at $x$ composition of the alloy, calculated in VCA, $\Delta V$-cation pseudopotential difference (Fig .2).

Experiments indicated that the bond lengths of the alloy components differ and stay almost the same as in pure compounds (Fig. 3). Dependence of the bond length $R_{A N}(x)$ and $R_{B N}(x)$ in $A_{1-x} B_{x} N$ can be characterized by dimensionless relaxation parameter [15]:

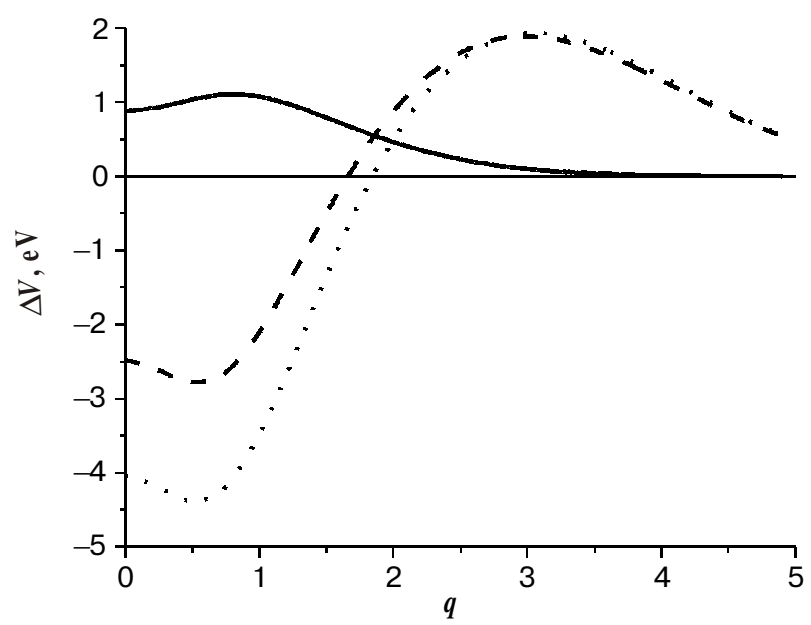

Fig. 2. Pseudopotential differences between $\mathrm{Ga}$ and $\mathrm{Al}$ atoms (solid line), Ga and In (dashed line), Al and In (dotted line).

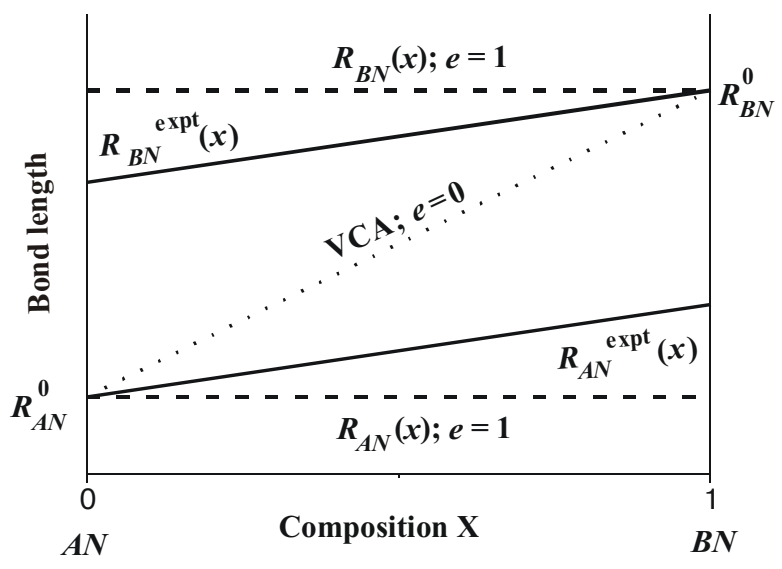

Fig. 3. Schematic variation of the bond length $R_{A N}(x)$ and $R_{B N}(x)$ in an $A_{1-x} B_{x} N$ alloy with composition. 
$e=\left(R_{B N}[A N: B]-R_{A N}{ }^{0}\right) /\left(R_{B N}{ }^{0}-R_{A N}{ }^{0}\right)$,

where $R_{B N}[A N: B]-B N$ bond length around the $B$ impurity in $A N$ crystal, $R_{B N}{ }^{0}, R_{A N}{ }^{0}$ - bondlengths of pure compounds.

On the basis of valence force field method calculations the dependence of the relaxation parameter $e$ on the alloy compounds properties was obtained [15]:

$e \approx 1 /\left[1+\left(\frac{R_{B N}^{0}}{R_{A N}^{0}}\right)^{3}\left(\frac{2}{3}-\frac{1}{2} f_{i}\right)\right]$

where $f_{i}$ - Philips's ionicity. It was also shown that the value of the bond relaxation can differ for alloy components leading to lattice constant bowing which is confirmed by first-principles calculations [16].

In this work the positional disorder was considered using the deformation dependent pseudopotentials [7] and by taking into account the deviations of the lattice constant from Vegard's rule. The value of the local deformation in an alloy was calculated relatively to the atomic position in pure compounds.

\section{Results and discussion}

Alloying effects cause the bowing in the band gap compositional dependence:

$E_{g}(x)=E_{g 1}+\left(E_{g 2}-E_{g 1}\right) x-b x(1-x)$

To analyze the physical origins of bowing in III-nitrides solid solutions we decompose $b$ into four components:

$b=b_{C D}+b_{S R}+b_{V D}+b_{C E}$

where $b_{C D}$ is a bowing due to compositional disorder, $b_{S R}$ connected with structure relaxation effect, $b_{V D}-$ bowing due to volume deformation effect and $b_{C E}$ - bowing due to charge exchange effect.

Table 3. Bowing and it's components.
Results of calculations are presented in Table 3. The calculations of the corrections due to compositional disorder (7) show that chemical disorder pushes the valence bands $\Gamma_{1 \mathrm{~V}}, \Gamma_{6 \mathrm{~V}}$ up and the conduction band $\Gamma_{1 \mathrm{C}}$ down, the largest effect being that on the valence band edge. Therefore the shrinkage of the VCA band gap results leading to a positive bowing $b_{C D}$. Table 3 shows that the values of the bowing are proportional to the pseudopotential differences of the cations (Fig. 2). Our results qualitatively agree with the results for other III-V compounds [10, 14], which also predict the shrinkage of the band gap.

The change of lattice constants of alloy components leads to a shift of the energy levels corresponding to the value of deformation potential (6). As the deformation potentials of AlN, GaN and InN differ (Table 4), the energy levels positions of the alloy will differ from the averaged values of pure compounds thus leading to bowing. The value of this bowing $b_{V D}$ is proportional to deformation potentials difference and to relative change of the lattice constant (Table 4):

$b_{V D} \sim \Delta a / a \cdot \Delta a_{g}$

The mixing of pure compounds into an alloy results in charge exchange between bonds. The charge flows from the less ionic bond to the more ionic and some extra charge accumulates on the weaker bond [17]. In VCA calculations this is revealed by the fact that the charge distribution along the bond in the alloy differs from averaged distribution in pure compounds leading to additional bowing $b_{C E}$.

Fig. 4 shows the valence charge distribution in pure III-nitrides. InN have the largest charge density around the anion, also the maximum of the charge density in it is the most shifted towards the anion which corresponds to it's largest ionicity in a III-nitrides series. One can see from Fig. 4 and Table 4 that the bigger is the difference between charge distribution of the alloy components the more noticeable is the bowing. For a qualitative characterization of charge redistribution we analyze the chemical bonding of III-nitrides in different models.

\begin{tabular}{l|l|l|l|l|l|l|l|l|l|l}
\hline \hline & $\Delta a_{g}, \mathrm{eV}$ & $\Delta a / a, \%$ & $\Delta g$ & $\Delta f_{i}$ & $b_{C D}, \mathrm{eV}$ & $b_{V D}, \mathrm{eV}$ & $b_{C E}, \mathrm{eV}$ & $b_{S R}, \mathrm{eV}$ & $b_{\text {total }}, \mathrm{eV}$ & $b_{\text {expt }}, \mathrm{eV}$ \\
\hline AlGaN & 1.1 & 2.5 & 0.09 & 0.051 & 0.19 & 0.176 & 0.199 & -0.262 & 0.3 & $0.6-1[24]$ \\
\hline InGaN & 4.3 & 10 & 0.12 & 0.078 & 1.23 & 2.471 & -1.531 & -0.286 & 1.89 & $2.5[25]$ \\
\hline AlInN & 5.4 & 12.5 & 0.03 & 0.129 & 2.575 & 4.37 & -2.49 & -0.841 & 3.62 & $1.3-6.9[26]$ \\
\hline \hline
\end{tabular}

Table 4. Parameters of the III-nitrides: lattice constant $a$, band gap $E_{g}$, deformation potential $a_{g}$, Philips's ionicity $f_{i}$, polarity $a_{p}$, charge asymmetry coefficient $g$.

\begin{tabular}{|c|c|c|c|c|c|c|c|c|c|c|c|}
\hline & $a, \AA$ & $E_{g}, \mathrm{eV}$ & $a_{g}, \mathrm{eV}$ & \multicolumn{2}{|c|}{$f_{i}$} & \multicolumn{4}{|c|}{$a_{p}$} & \multicolumn{2}{|c|}{$g$} \\
\hline & & & & [5] & [22] & [23] & [20] & [12] & This work & [5] & This work \\
\hline$\overline{\mathrm{AlN}}$ & 3.11 & 6.23 & -9 & 0.449 & 0.36 & - & 1.43 & - & 0.85 & 0.794 & 0.61 \\
\hline $\mathrm{GaN}$ & 3.19 & 3.5 & -8.2 & 0.500 & 0.37 & 0.46 & 0.67 & 0.74 & 0.98 & 0.780 & 0.52 \\
\hline $\mathrm{InN}$ & 3.53 & 0.7 & -4 & 0.578 & 0.49 & 0.39 & 0.3 & 0.81 & 1.17 & 0.853 & 0.64 \\
\hline
\end{tabular}




\section{O.V. Voznyy, V.G. Deibuk: Chemical bonding and optical bowing in III-nitrides ...}

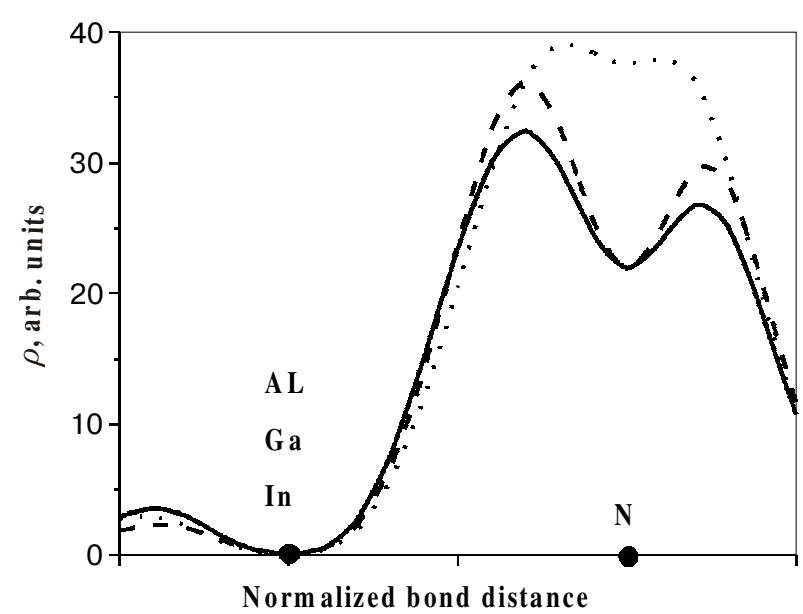

Fig. 4. Valence charge density of binary compounds along [0001] bond: GaN - solid line, AlN - dashed line, InN - dotted line.

Comparing Fig. 2 and Fig. 4 one can see that the difference of the charge density along the bond corresponds to the pseudopotential difference of the cations so we can use polarity $a_{p}$ to describe it. But for binary compounds $\mathrm{AlN}, \mathrm{GaN}, \mathrm{InN}$ the values of polarity are very controversial (Table 4) moreover polarity depicts the asymmetry of the pseudopotential only in one point of the reciprocal space. These facts make polarity not suitable for characterization of the chemical bonding in IIInitrides.

Charge asymmetry coefficient $g[5]$ was successfully used to describe the physical properties of tetrahedral III-V alloys [18]. During the fitting procedure for the pseudopotentials parameters [7] it was noted that for each alloy the value of bowing $b$ depends on the difference of the charge asymmetry coefficients, which implicitly depend on the valence band width. But there is no uniquely determined rule for all investigated alloys. Table 4 shows that Philips's ionicity $f_{i}$ increases with the charge number of the cation. For the charge asymmetry coefficient in contrast to other III-V compounds [18] this is not the case which agrees with other calculations [5]. So, despite that charge asymmetry coefficient is the fullest integral characteristics of the charge distribution, the Philips's ionicity describes the changes in chemical bonding better. We can suppose that charge distribution along the bond is more important for chemical bonding properties than integral charge distribution over all unit cell.

Bond lengths and angles relaxation leads to shift of the atomic positions from the ones given by Vegard's rule and this also leads to charge redistribution. As the bond lengths relax back to the initial values in the pure compounds this effect is inverse to volume deformation so the value of bowing $b_{S R}$ is negative (Table 3 ). Different values of the relaxation parameters [10] of III-nitrides alloy compounds leads to bowing of lattice constant with a value of about $0.3 \%$. It is important to take this bowing into account because the value of the lattice constant is used to determine the composition of the alloy experimentally.

Our results are in good agreement with other calculations that investigated the compositional and positional disorder using larger elementary cells with 8 and 16 atoms [19] and large clusters with thousands of atoms [16]. First-principles calculations [16] showed that small unit cells describe random alloys satisfactorily because for many physical properties interactions between distant neighbours are generally much less important that those between close neighbours.

\section{Conclusions}

We have shown that the chemical bonding in III-nitrides alloys is correctly described by Philips's ionicity while there is no unambiguous connection between charge asymmetry model and chemical bonding properties. The influence of alloying effects on optical bowing is analyzed. The bowing is mainly caused by compositional disorder and volume deformation effects followed by chemical charge-transfer contribution. The connection of the bowing value with the basic parameters of the materials, such as deformation potential, lattice constant and ionicity, is derived, which allows evaluating the bowing for other materials.

\section{References}

1. H. Markoc Nitride semiconductors and devices. - Berlin: Springer Verlag (1999).

2. S. Nakamura Introduction to nitride semiconductor blue lasers and light emitting diodes. - London: Taylor and Francis (2000).

3. J. S. Philips Bonds and bands in semiconductors. - New York: Acad. Press (1973).

4. P. Vogl, Dynamical effective charges in semiconductors: a pseudopotential approach // J.Phys.C. 11, pp.251 (1978).

5. A. Garcia, M. L. Cohen, First-principles ionicity scales. Charge asymmetry in the solid state // Phys.Rev.B. 47 (8), pp.4215-4220 (1993).

6. V. Deibuk., A. Vozny, M. Sletov, Electronic band structure and spatial charge distribution of GaAlN alloys // Semiconductors 34 (1), pp.35-39 (2000).

7. V. Deibuk, A. Vozny, M. Sletov, A. Sletov, Features of Optical Properties of AlGaN Solid Solutions // Semiconductors 36 (4), pp.398-403 (2001).

8. T. Matilla, L.-W. Wang, A. Zunger, Electronic consequences of lateral composition modulation in semiconductor alloys // Phys.Rev. B 59, pp.15270 (1999).

9. D. R. Hamman, Generalized norm-conserving pseudopotentials // Phys.Rev.B 40, pp.2980-2987 (1989).

10. G. Bohm, K. Unger, Selfconsistent calculation of disorderinduced corrections to the VCA band-strucures of AlGaAs and AlGaP // Phys.Stat.Sol.B 216, pp.961-973 (1999).

11. I. Vurgaftman, J. Meyer, Band parameters for III-V compound semiconductors and their alloys // J. Appl. Phys. 89, pp.5815-5874 (2001).

12. Y. Yeo, T. Chong, M. Li, Electronic band structure and effective-mass parameters of wurtzite $\mathrm{GaN}$ and $\mathrm{InN} / / \mathrm{J}$. Appl. Phys. 83 (3), pp.1429-1436 (1998).

13. D. Chadi, M. L. Cohen, Special points in the Brillouin zone // Phys. Rev. B 8, pp.5747 (1973). 


\section{O.V. Voznyy, V.G. Deibuk: Chemical bonding and optical bowing in III-nitrides ...}

14. A. Baldareschi, K. Maschke, Band structure of semiconductor alloys beyond the virtual crystal approximation. Effect of compositional disorder on the energy gaps in GaPAs // Sol.St.Comm. 16, pp.99-102 (1975).

15. J. L. Martins, A. Zunger, Bond length around isovalent impurities and in semiconductor solid solutions // Phys. Rev. B 30 (10), - pp.6217-6220 (1984).

16. K. Kim, W. R. L. Lambrecht, B. Segall, Theoretical study of group-III nitride alloys // Phys. Rev. B 53, pp.16310 (2001).

17. G. Srivastava, J. Martins, A. Zunger, Atomic structure and ordering in semiconductor alloys // Phys.Rev.B 31 (4), pp.2561-2564 (1985).

18. V. Deibuk, Y. Viklyuk, Chemical bondong and elastic constants of some ternary AIIIBV solid solutions // Semiconductors 36 (10), pp.1171 (2002).

19. A. Zaoui, Density functional theory study on the origins of the gap bowing in $\mathrm{Zn}_{1-\mathrm{x}} \mathrm{Mg}_{\mathrm{x}} \mathrm{Se} / /$ J.Phys. Condens. Matter 14 pp.4025-4033 (2002).

20. S. Pugh, D. Dugdale, S. Brand, R. Abram, Electronic structure calculations on nitride semiconductors // Semicond. Sci. Technol. 14, pp.23-31 (1999).
21. A. Rubio, J. L. Corkill, M. L. Cohen, et.al. Quasiparticle band structure of AlN and GaN // Phys. Rev. B 48 (16), pp.11810-11816 (1993)

22. S. Davydov, Estimation of III-group nitrides parameters: BN, AlN, GaN and InN // Semiconductors 36 (1), pp.45 (2002).

23. N. Bouarissa, K. Kassali, Mechanical Properties and Elastic Constants of Zinc-Blende $\mathrm{Ga}_{1-\mathrm{x}} \mathrm{In}_{\mathrm{x}} \mathrm{N}$ Alloys // Phys. Stat. Sol. B 228 (3), pp. 663-670 (2001).

24. M. Leroux, S. Dalmasso et. al. Optical characterization of $\mathrm{Al}_{\mathrm{x}} \mathrm{Ga}_{1-\mathrm{x}} \mathrm{N}$ alloys $(\mathrm{x}<0.7)$ grown on sapphire or silicon // Phys. Stat. Sol. B 234 (3), pp.887-891 (2002).

25. V. Davydov, A. Klochikhin et.al. Band gap of hexagonal InN and InGaN alloys // Phys. Stat. Sol. B 234 (3), pp.787795 (2002).

26. L. Teles, L. Scolfaro et.al. Phase separation, gap bowing, and structural properties of cubic $\operatorname{In}_{\mathrm{x}} \mathrm{Al}_{1-\mathrm{x}} \mathrm{N} / /$ Phys. Stat. Sol. B 234 (3), pp.956-960 (2002). 